\title{
Tripartite Motif Containing 24 Acts as a Novel Coactivator of the Constitutive Active/Androstane Receptor ${ }^{\mathbb{\S}}$
}

\author{
Yuichiro Kanno, Yuki Kure, Saori Kobayashi, Mariko Mizuno, Yumi Tsuchiya, Naoya Yamashita, \\ Kiyomitsu Nemoto, and Yoshio Inouye
}

Department of Molecular Toxicology, Faculty of Pharmaceutical Sciences, Toho University, Funabashi, Chiba, Japan

Received July 20, 2017; accepted October 30, 2017

\begin{abstract}
The constitutive androstane receptor (CAR) is a nuclear receptor that acts as a transcription factor for a variety of genes, including genes encoding xenobiotic, steroid, and drug-metabolizing enzymes and transporters. Transactivation of a target gene by a transcription factor is generally mediated through the concerted and stepwise recruitment of various proteins termed coregulators, including coactivators and corepressors. In this study, TRIM24 (also known as transcriptional intermediary factor 1 alpha) was found to interact with the CAR. TRIM24 enhanced the CAR-dependent transactivation in reporter assays using the direct repeat- 4 motif, a binding site of the CAR. This
\end{abstract}

enhancement was synergistically augmented in the presence of steroid receptor coactivator (SRC) 1 or SRC2, both of which are coactivators of the CAR. In addition, TRIM24 was recruited to the CAR-binding element of the CYP2B6 promoter together with the CAR. We also noted that knockdown of TRIM24 suppressed CAR-induced CYP2B6 mRNA expression in HepTR/CAR and HepaRG cells and suppressed CAR-induced CYP3A4 mRNA expression in HepaRG cells but not HepTR/CAR cells. From these results, we suggest that TRIM24 is a novel coactivator of the CAR that is involved in cell- and/or promoter- selective transactivation.

\section{Introduction}

The nuclear receptor (NR) superfamily comprises a large superfamily of transcription factors, many of which regulate gene expression in a ligand-dependent manner (Mangelsdorf et al., 1995; Gronemeyer et al., 2004). Members of the NR superfamily are composed of several domains, including an $\mathrm{N}$-terminal trans-activation domain containing activation function 1, a DNA-binding domain (DBD) containing zinc fingers that bind to specific DNA sequences, a hinge region, and a ligand-binding domain (LBD) containing ligand-dependent activation function 2. The transactivation ability of a NR is generally mediated through the concerted and stepwise recruitment of various proteins termed coregulators, including coactivators and corepressors.

The constitutive androstane receptor [(CAR) NR1I3], a member of the NR superfamily, plays a key role in cellular responses to xenochemical stimuli by inducing the gene expression of various xenobioticmetabolizing enzymes and transporters. Its typical targets are the CYP2B, 2C, and 3A subfamilies and UGT1A1 (Honkakoski et al., 1998; Sueyoshi et al., 1999; Sugatani et al., 2001). The CAR is predominantly localized in the cytoplasm of hepatocytes in vivo and in primary hepatocytes, and is translocated to the nucleus in response to the binding of ligands or indirect activators, such as 6-(4-chlorophenyl)imidazo[2,1-b][1,3]thiazole-5carbaldehyde $O$-(3,4-dichlorobenzyl)oxime (CITCO) and phenobarbital,

This work was supported by the Japan Society for the Promotion of Science (JSPS), Grant-in-Aid for Scientific Research (KAKENHI) [Grants 23790104 and 25460175].

https://doi.org/10.1124/dmd.117.077693.

S This article has supplemental material available at dmd.aspetjournals.org.

respectively. Following nuclear translocation, the CAR forms a heterodimer with the retinoid $\mathrm{X}$ receptor $\alpha$ and binds to specific sequences (responsive elements) in the promoter regions of target genes (Baes et al., 1994). Unlike other NRs, the CAR has constitutive transcriptional activity. One reason for this is that the CAR is able to recruit coactivators without binding to ligands. Some coactivators that interact with the CAR have been identified, such as steroid receptor coactivator (SRC) 1 (Muangmoonchai et al., 2001), SRC2 (also known as transcriptional intermediary factor 2) (Min et al., 2002), SRC3 (Chen et al., 2012), NCoA6 (Surapureddi et al., 2008), activating signal cointegrator 2 (Choi et al., 2005), GADD45 beta (Tian et al., 2011) and PGC1a (Shiraki et al., 2003). We have also previously identified two coactivators of the CAR, DEAD box DNA/RNA helicase DP97 (Kanno et al., 2012) and protein arginine methyltransferase 5 (Kanno et al., 2015). However, the mechanisms by which the CAR regulates gene expression are still being elucidated.

The aim of this study was to discover novel cofactor(s) involved in the mechanisms underlying CAR-mediated gene regulation. To identify novel cofactors, we performed a coimmunoprecipitation analysis using a human hepatocellular carcinoma hepG2 cells expressing FLAG-epitope tagged CAR (FLAG-CAR). Among the coprecipitated proteins, we focused on TRIM24 as a novel CAR-interacting protein.

\section{Materials and Methods}

Plasmid Construction and Chemicals. The construction of expression plasmids encoding N-terminal myc or FLAG epitope-tagged human CAR proteins (myc-CAR or FLAG-CAR) has been reported previously (Kanno et al., 2005, 2007, 2012). The expression plasmid encoding a GAL4DBD/CAR-LBD fusion protein was constructed as reported previously (Kanno and Inouye, 2010). The pEBMultipuro/FLAG-CAR plasmid was constructed by

ABBREVIATIONS: CAR, constitutive androstane receptor; ChIP, chromatin immunoprecipitation; CITCO, 6-(4-chlorophenyl)imidazo[2,1-b] $[1,3]$ thiazole-5-carbaldehyde O-(3,4-dichlorobenzyl)oxime; DBD, DNA-binding domain; DR4, direct repeat-4; LBD, ligand-binding domain; NR, nuclear receptor; PBREM, phenobarbital responsive enhancer module; PCR, polymerase chain reaction; siRNA, small interfering RNA; SRC, steroid receptor coactivator; Tet, tetracycline. 


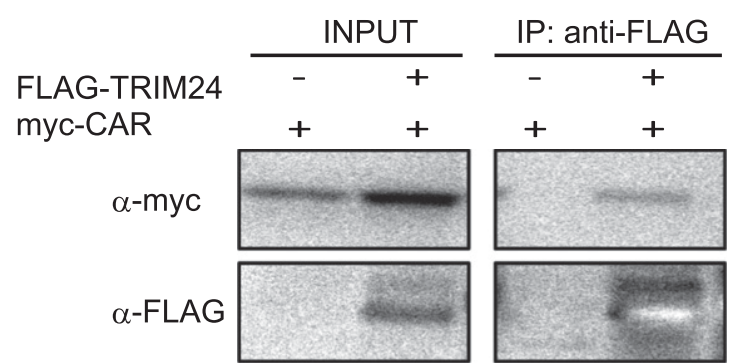

Fig. 1. TRIM24 interacts with the CAR. HEK293 cells were transfected with expression plasmids for myc-CAR and/or FLAG-TRIM24, or with an empty plasmid, as indicated. Whole cell lysates prepared from transfected cells were immunoprecipitated using anti-DDDDK-tag mAb-magnetic beads. Coprecipitants were resolved by SDS-PAGE and detected by western blot analyses using anti-myc $(\alpha$-myc) and anti-DDDDK ( $\alpha$-FLAG) antibodies. The data show one of two independent experiments.

inserting a FLAG-epitope tagged human CAR fragment into the multiple cloning site of pEBMultipuro (Wako, Osaka, Japan), which carries Epstein-Barr virus nuclear antigen 1 to allow episomal replication of the plasmid without integration into the host genome. It also included the puromycin $N$-acetyltransferase gene to enable selection of transfectants by puromycin. Full-length human TRIM24 cDNA was amplified by reverse transcription polymerase chain reaction (PCR) using mRNA prepared from HepG2 cells. The expression plasmid encoding FLAG epitope-tagged TRIM24 (FLAG-TRIM24) was constructed based on the pCMV-3Tag-6 vector (Stratagene, Santa Clara, CA) using an In-Fusion HD Cloning Kit (Takara Bio, Shiga, Japan). The direct repeat-4 (DR4)-driven luciferase reporter plasmid (pDR4-Luc) was constructed by inserting three tandem repeats of the DR4 motif sequence (AGTTCATGGCAGTTCA), into pGL4.24 (Promega, Madison, WI), which has a minimal promoter (Kanno et al., 2012). The GAL-driven luciferase reporter (pG5-luc) was purchased from Promega. The expression plasmids encoding small interfering RNA (siRNA) to knock down TRIM24 expression were generated by inserting the following shTRIM24-\#1 or shTRIM24-\#2 annealed double-strand fragments into the BamHI-HindIII sites of pBAsi-hU6 Pur, which contains an RNA polymerase III promoter for the expression of siRNA (Takara Bio, Shiga, Japan). shTRIM24-\#1: 5'-GAT CCG CAT GAA ATG AGC CTG GCT TTC TCG AGA AAG CCA GGC TCA TTT CAT GCT TTT TA- ${ }^{\prime}$ ' and 5' AGC TTA AAA AG C ATG AAA TGA GCC TGG CTT TCT CGA GAA AGC CAG GCT CAT TTC ATG CG-3'; and shTRIM24-\#2: 5'-GAT CCG CCA TGA AAT GAG CCT GGC TTT CTC GAG AAA GCC AGG CTC ATT TCA TGG CTT TTT A-3' and 5'-AGC TTA AAA AGC CAT GAA ATG AGC CTG GCT
TTC TCG AGA AAG CCA GGC TCA TTT CAT GGC G-3' . Tetracycline (Tet), PK11195, CITCO, and dimethylsulfoxide were purchased from Sigma-Aldrich (St. Louis, MO).

Cell Lines and Cell Culture. The HepTR/hCAR cell line, derived from HepG2 cells, was previously established using the T-REx system (Invitrogen, Carlsbad, CA), in which the expression of hCAR can be induced by Tet treatment (Kanno et al., 2012). HEK293, MCF-7, HepG2, and HepTR/hCAR cells were cultured in Dulbecco's modified Eagle's medium (Wako) containing $10 \%$ fetal bovine serum and penicillin-streptomycin at $37^{\circ} \mathrm{C}$ in a humidified atmosphere containing $5 \% \mathrm{CO}_{2}$. Differentiated HepaRG cells (KAC, Kyoto, Japan) were maintained in Williams' medium E (Life Technologies, Grand Island, NY) supplemented with $10 \%$ fetal bovine serum, $5 \mu \mathrm{g} / \mathrm{ml}$ insulin, and $50 \mu \mathrm{M}$ hydrocortisone (WAKO, Osaka, Japan) at $37^{\circ} \mathrm{C}$ under $5 \% \mathrm{CO}_{2}$ and $95 \%$ air according to the manufacturer's instructions.

Identification of TRIM24 by Coimmunoprecipitation. pEBMultipuro/ FLAG-hCAR was transfected into HepG2 cells, and then a clone stably expressing FLAG-hCAR was isolated by puromycin selection, named fCAR cells. The clone cells were harvested and suspended in lysis buffer [50 mM Tris$\mathrm{HCl}$ (pH 7.5), 1 mM EDTA, $150 \mathrm{mM} \mathrm{NaCl}$, and 1\% Triton X-100] containing a protease inhibitor cocktail (Roche Diagnostics, Mannheim, Germany). The recovered supernatant was incubated with Anti-DDDDK-tag mAb-magnetic beads (MBL, Aichi, Japan), which are equivalent to anti-FLAG-tag monoclonal antibodymagnetic beads, at $4^{\circ} \mathrm{C}$ for 4 hours to concentrate the proteins coimmunoprecipitated with FLAG-hCAR. Coimmunoprecipitated proteins were identified using a mass spectrometry service provided by Oncomics (Nagoya, Japan). Among the candidate proteins interacting with the CAR, we decided to further analyze the properties of TRIM24.

Coimmunoprecipitation using HEK293 cells was also performed to confirm that TRIM24 can interact with the CAR. HEK293 cells were seeded in 60-mm plates and transfected with expression plasmids for myc-hCAR and/or FLAGTRIM24 using the PEI Max reagent (Polysciences, Warrington, PA). At 48 hours post-transfection, cells were harvested after being washed twice with ice-cold phosphate-buffered saline and were suspended in lysis buffer containing a protease inhibitor cocktail (Roche Diagnostics). The supernatants were incubated with Anti-DDDDK-tag mAb-magnetic beads at $4^{\circ} \mathrm{C}$ for 4 hours. The beads were washed three times with wash buffer [50 mM Tris- $\mathrm{HCl}$ (pH 7.5), 1 mM EDTA, $650 \mathrm{mM} \mathrm{NaCl}$, and $0.1 \%$ Triton X-100], and then suspended in sodium dodecyl sulfate sample buffer [10\% glycerol, $2 \%$ SDS, $62.5 \mathrm{mM}$ Tris- $\mathrm{HCl}$ (pH 6.8), and $1 \mathrm{mM}$ DTT]. The coimmunoprecipitated proteins were then separated by SDSPAGE and detected by western blotting using anti-myc-tag- and anti-DDDDK (FLAG)-tag-HRP-DirecT antibodies. The signals were captured by a LuminoGraph II (ATTO, Tokyo, Japan).
A

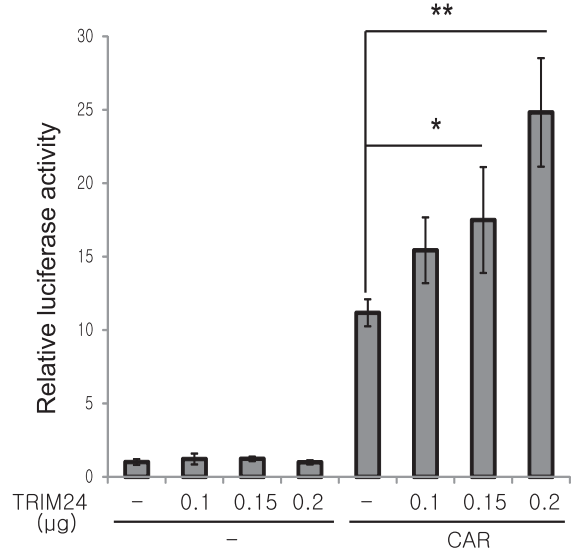

B

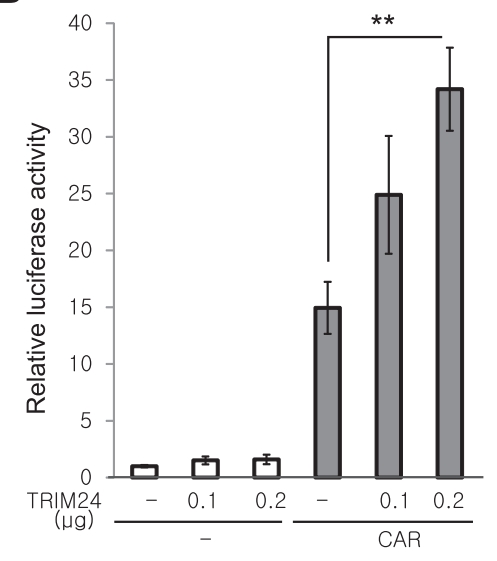

C

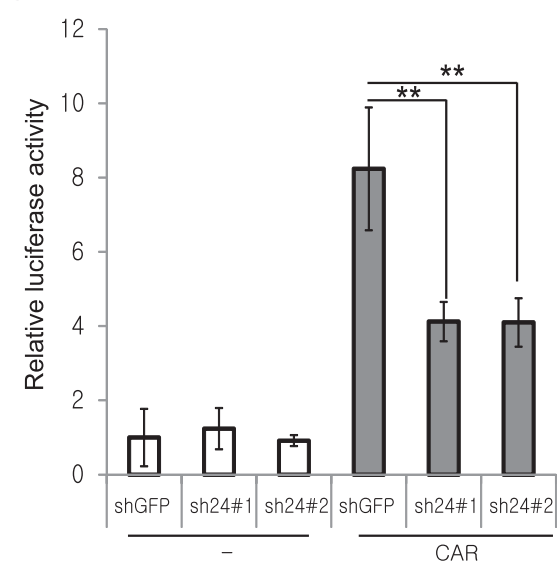

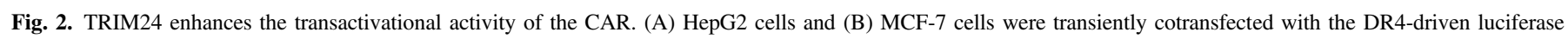

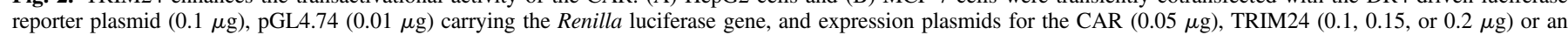

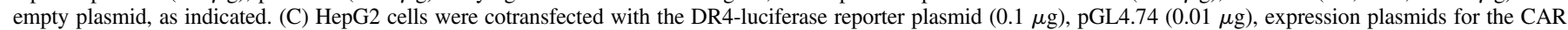

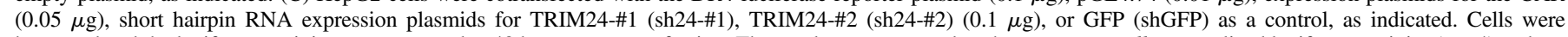

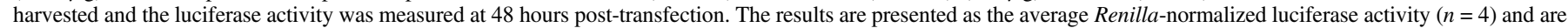
expressed as the mean \pm S.D. $\left(* * P<0.01 ;{ }^{*} P<0.05\right)$. The graph presents one of three independent experiments. 


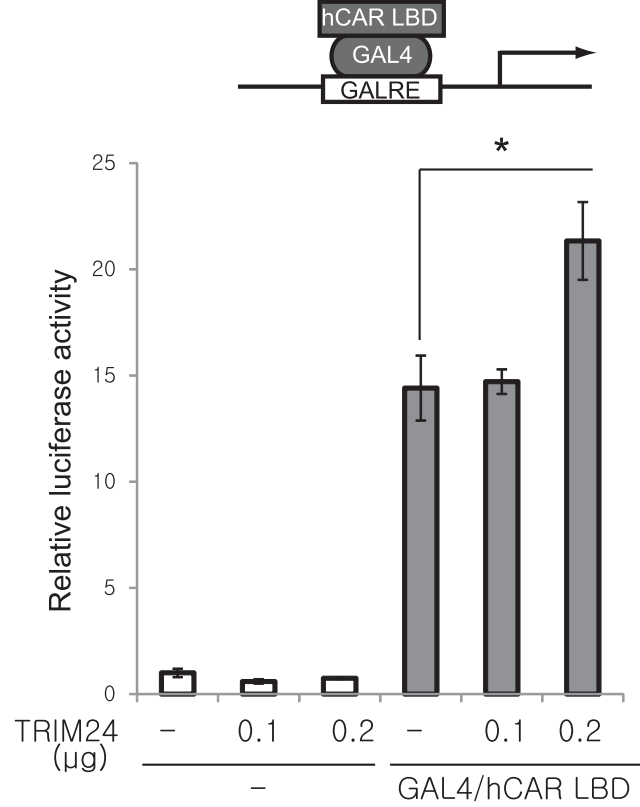

Fig. 3. TRIM 24 enhances the transactivation activity of the CAR through the LBD of the CAR. HepG2 cells were cotransfected with the GAL responsive element (GALRE)/luciferase reporter plasmid $(0.1 \mu \mathrm{g})$ carrying the GALresponsive element and pGL4.74 $(0.01 \mu \mathrm{g})$ and expression plasmids for the GAL4-DBD/CAR-LBD fusion protein $(0.05 \mu \mathrm{g})$, TRIM24 $(0.1$ or $0.2 \mu \mathrm{g})$, or an empty plasmid, as indicated. Cells were harvested and the luciferase activity was measured at 48 hours post-transfection. The results are presented as the average of Renilla-normalized luciferase activity $(n=4)$ and are expressed as the mean \pm S.D. $(* P<0.05)$. The graph presents the results of one of three independent experiments.

Luciferase Reporter Assay. The appropriate expression plasmids, reporter plasmids, and pGL4.74 (hRluc/TK; Promega) carrying the Renilla luciferase gene as an internal standard were transfected into HepG2 cells using PEI Max Reagent (Polysciences). After 48 hours, the luciferase activity was measured using the Dual-Luciferase Reporter Assay System (Promega). The activities of firefly luciferase were normalized to those of Renilla luciferase.

TRIM24 Knockdown and Quantitative Real-Time PCR. HepTR/hCAR and HepaRG cells were transfected with TRIM24-targeting or control siRNA (Sigma Aldrich, St Louis, MO) using Lipofectamine RNAiMAX Reagent (Invitrogen) according to the manufacturer's instructions. After 48 hours, total RNA was isolated using ISOGEN II (Nippon Gene, Tokyo, Japan) and cDNA was synthesized using a ReverTraAce qPCR RT kit (Toyobo, Osaka, Japan). Quantitative PCR was performed using the KOD SYBR qPCR mix (Toyobo, Osaka, Japan) according to the manufacturer's protocol on a 7500 Fast system (Applied Biosystems, Foster City, CA). The specific PCR primers used were as follows: $C Y P 2 B 6$ ( $5^{\prime}$-AAG CGG ATT TGT CTT GGT GAA$3^{\prime}$ and $5^{\prime}$-TGG AGG ATG GTG GTG AAG AAG-3'), CYP3A4 (5'-CCA AGC TAT GCT CTT CAC CG-3' and $5^{\prime}$-TCA GGC TCC ACT TAC GGT GC-3'), UGT1A1 (5'-AGT GGA TGG CAG CCA CTG GCT-3' and 5'-CAG TAA GTG GGA ACA GCC AGA-3'), CAR (5'-CCAGCTCATCTGTTCATCCA-3' and 5'GGTAACTCCAGGTCGGTCAG- $\left.3^{\prime}\right)$, and $\beta$-actin ( $5^{\prime}$-TCC TCCTGA GCG CAA

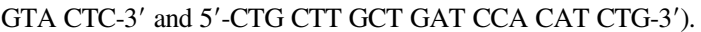

Chromatin Immunoprecipitation (ChIP) Assays. ChIP assays were performed using a Simple ChIP Enzymatic Chromatin IP kit (Cell Signaling Technology, Danvers, MA). Immunoprecipitation was performed using antibodies directed against the CAR (N4111; Perceus Proteomics Inc., Tokyo, Japan) or TRIM24 (14208; Proteintech, Chicago, IL), or samples were incubated with normal IgG (Cell Signaling Technology) as a negative control. After immunoprecipitation, DNA fragments were subjected to quantitative PCR using primers containing the phenobarbital responsive enhancer module (PBREM) of the CYP2B6 promoter (forward 5' - CTG CAA TGA GCA CCC AAT CTT - 3 ' and reverse $5^{\prime}$ - ACA CAT CCT CTG ACA GGG TCA -3').

Statistical Analysis. Statistical comparisons were performed with one-way analysis of variance followed by Dunnett's multiple comparisons test as the post hoc test, and differences were considered statistically significant at $* P<0.05$.

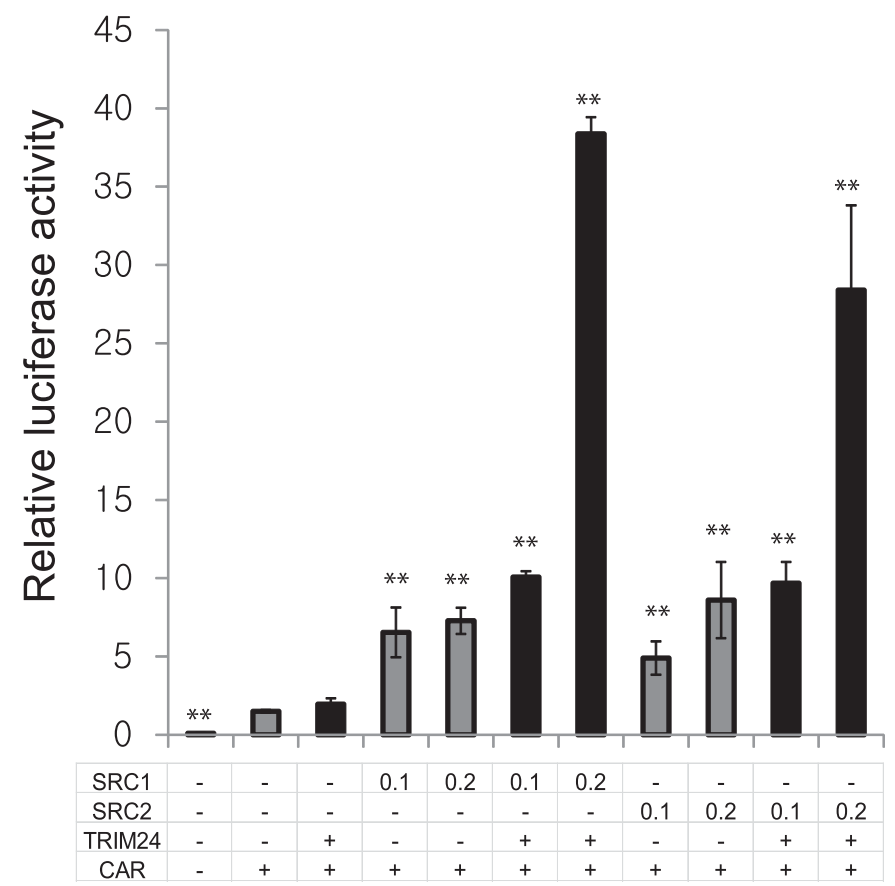

Fig. 4. TRIM24 synergistically enhances the CAR-mediated transactivation with other coactivators. HepG2 cells were cotransfected with the DR4-luciferase reporter plasmid $(0.1 \mu \mathrm{g})$, pGL4.74 $(0.01 \mu \mathrm{g})$, and expression plasmids for the CAR $(0.05 \mu \mathrm{g})$, TRIM24 $(0.1 \mu \mathrm{g}$ each), SRC1 and/or SRC2 (0.1 or $0.2 \mu \mathrm{g})$, or an empty plasmid, as indicated. Cells were harvested and the luciferase activity was measured at 48 hours post-transfection. The results are presented as the average of the Renilla-normalized luciferase activity $(n=4)$ and are expressed as the mean \pm S.D. ( $* * P<0.01$ vs. CAR-mediated transactivation, column 2). The graph presents the data for one of three independent experiments.

\section{Results}

TRIM24 Was Identified as a CAR-Interacting Protein. To identify CAR-interacting proteins, we established stably $\mathrm{N}$-terminal FLAG-tagged CAR expressing HepG2 cells (fCAR cells). CAR-protein

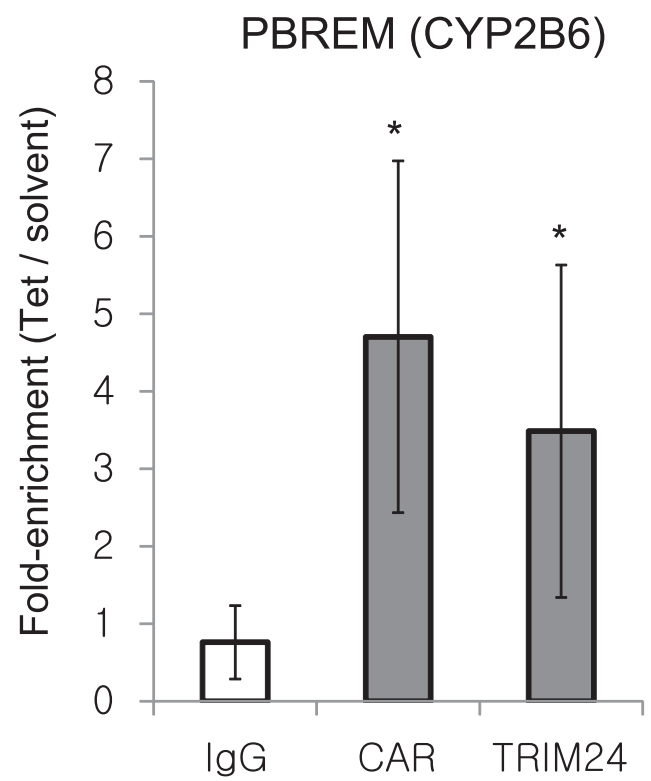

Fig. 5. TRIM24 is recruited to the PBREM of the CYP2B6 promoter together with the CAR. HepTR/hCAR cells were treated with Tet or solvent for 24 hours. Cells were subjected to ChIP with anti-CAR and TRIM24 antibodies and analyzed by quantitative PCR with primers flanking the PBREM of the CYP2B6 promoter. The results are presented as the average fold enrichment induced by Tet treatment $(n=4)$ and are expressed as the mean \pm S.D. Significant differences in recruitment by Tet treatment compared with $\operatorname{IgG}$ are indicated with an asterisk $\left({ }^{*} P<0.05\right)$. 


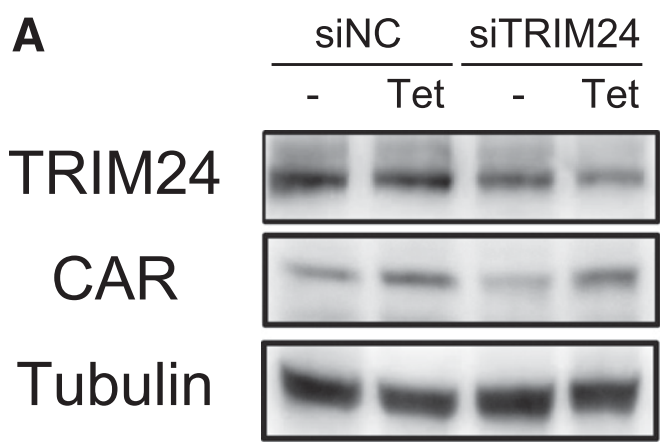

B

CYP2B6

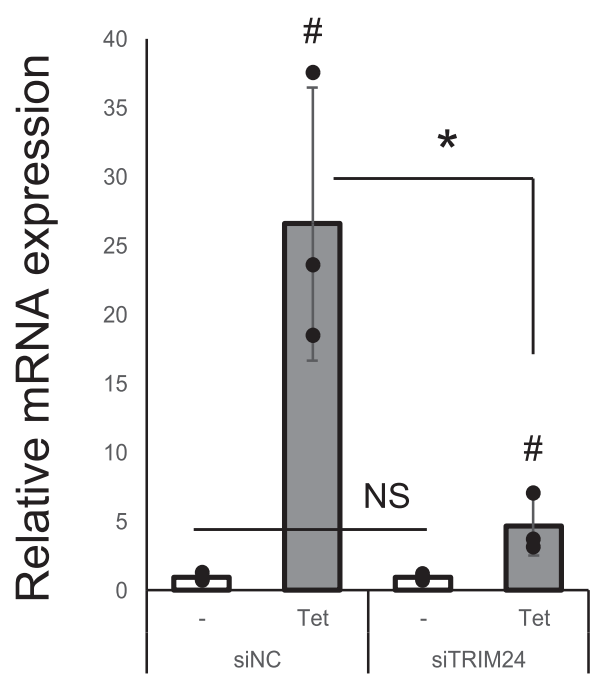

CYP3A4

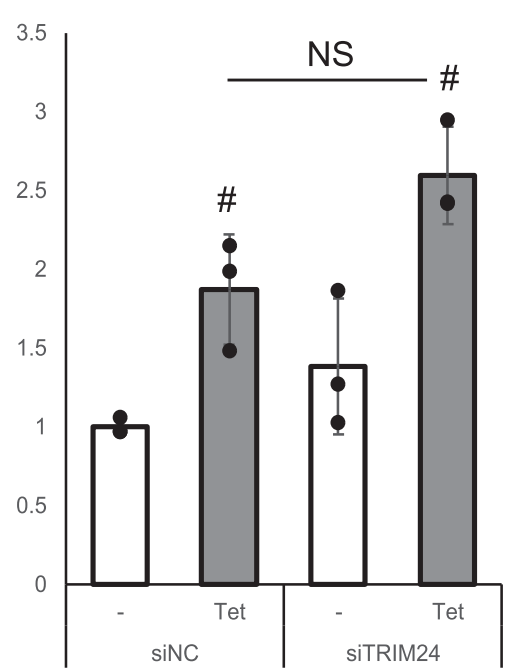

CAR

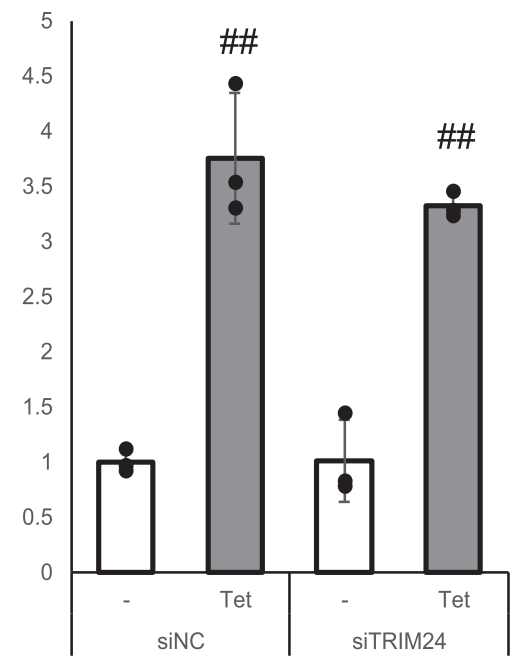

Fig. 6. TRIM24 knockdown suppresses CAR-regulated gene expression in HepTR/hCAR cells. HepTR/hCAR cells were treated with a TRIM24-targeting or control siRNA. Cells were then treated with Tet or solvent for 48 hours. (A) The protein expression levels of TRIM24 and the CAR were detected by western blotting. The data show one of two independent experiments. (B) The levels of CYP2B6 and CYP3A4 mRNA were measured by real-time quantitative PCR. The results were normalized to those for $\beta$-actin $(n=3)$. Individual values are shown along with the mean \pm S.D. $\left[{ }^{\#} P<0.05\right.$; ${ }^{\#} P<0.01$ vs. (-) siNC, $* P<0.05$ vs. (tet) siNC]. The graph presents one of three independent experiments.

complexes were immunoprecipitated with an anti-FLAG antibody from the lysates of fCAR cells. Immunoprecipitated proteins were analyzed by liquid chromatography-tandem mass spectrometry systems (Supplemental Material). Identified proteins contained previously reported CARinteracting proteins, such as CAR, Hsp90, and Hsp70 (Yoshinari et al., 2003; Timsit and Negishi, 2014). An analysis of the complexes obtained from these experiments identified TRIM24 as a novel candidate CARinteracting protein. TRIM24, also known as transcriptional intermediary factor $1 \alpha$, belongs to the tripartite motif family and contains a RING finger, two B-box zinc finger domains, and a coiled-coil region. TRIM24 has been reported to serve as a coregulator of several NRs, including the glucocorticoid, estrogen, androgen, retinoic acid, thyroid hormone, and vitamin D receptors (Le Douarin et al., 1995, 1996; vom Baur et al., 1996; Teyssier et al., 2006; Kikuchi et al., 2009).

To confirm the interaction of TRIM24 with the CAR, a coimmunoprecipitation assay was performed using lysates from a human embryonic kidney cell line, HEK293, expressing myc epitopetagged CAR (myc-CAR) and/or FLAG-epitope tagged TRIM24 (FLAG-TRIM24). As shown in Fig. 1, the anti-FLAG antibody immunoprecipitate from the lysates of cells expressing both myc-CAR and FLAG-TRIM24 reacted with the anti-myc antibody, confirming that TRIM24 interacts with the CAR.

TRIM24 Enhances CAR Transactivity. We next examined the role(s) of TRIM24 in CAR-mediated transcriptional regulation in HepG2 cells. It has previously been reported that transformed and immortalized human cells, such as HepG2 cells, lack the ability to retain the CAR in the cytoplasm (Kawamoto et al., 1999; Kanno et al., 2005). Thus, the CAR spontaneously accumulates in the nucleus without activator or ligand treatment. We found that the transfection of increasing amounts of either a TRIM24 or CAR expression plasmid increased the luciferase reporter activity controlled by the DR4 motif, a binding element of the CAR (Fig. 2A). Similar results were observed in other cell lines, including human breast cancer MCF-7 (Fig. 2B) and human embryonic kidney HEK293 cells (data not shown). We also evaluated the effects of TRIM24 knockdown on the transactivation of the CAR. A TRIM24-targeting short hairpin RNA (shTRIM24-\#1 or -\#2) expression plasmid, the DR4-driven luciferase reporter plasmid, and the CAR expression plasmid were cotransfected into HepG 2 cells and the luciferase activity was measured. The results showed that knocking down TRIM24 reduced the luciferase activity in cells expressing the CAR (Fig. 2C); further supporting that 


\section{HepaRG}

\section{CYP3A4}

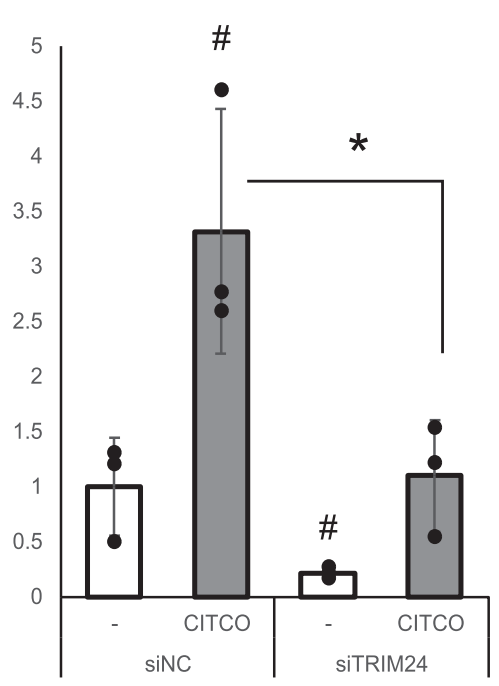

TRIM24

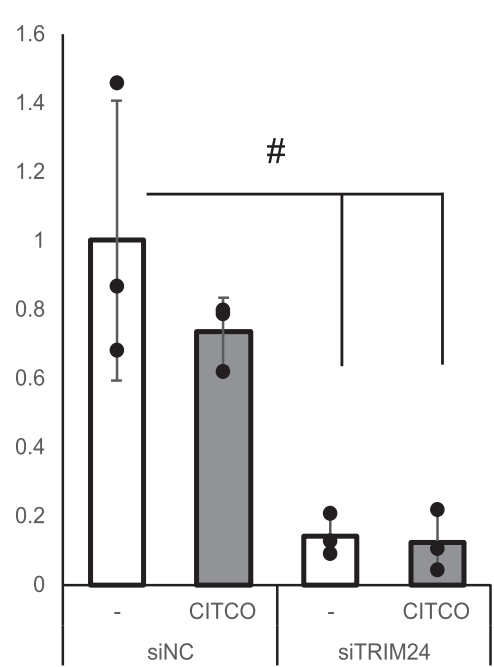

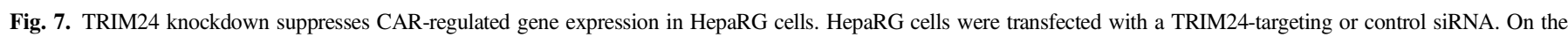

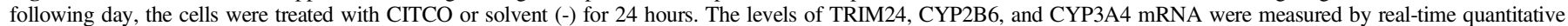

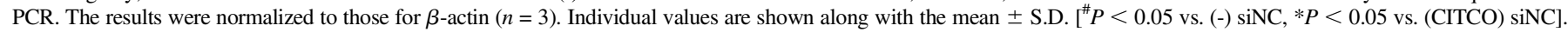

TRIM24 is an activator of the CAR. These results suggest that TRIM24 acts as coactivator of the CAR.

TRIM24 Enhances the Transactivity of CAR through the LBD of the CAR. The activation function 2 domain in the LBD of the CAR plays an essential role in the high constitutive activity of the CAR in the absence of ligand by facilitating the active conformation of the CAR and recruiting coactivators, such as SRC1 and SRC2 (Windshügel et al., 2005; Jyrkkärinne et al., 2012). Moreover, the GAL4 DBD-CAR LBD fusion protein (GAL4/CAR LBD) can activate transcription in response to the GAL responsive element without a CAR ligand in the mammalian two-hybrid system (Kanno and Inouye, 2010). In the present study, the significance of the CAR activation function 2 domain in the function of TRIM24 was evaluated using TRIM24 and GAL4 DBD/CAR LBD expression plasmids and a GAL responsive element/luciferase reporter plasmid. Overexpression of TRIM24 increased the luciferase activity (Fig. 3), suggesting that TRIM24 enhances CAR transactivity through the CAR LBD.

TRIM24-Mediated Enhancement of the CAR Transactivity Is Synergistically Augmented in the Presence of SRC1 or SRC2. Several studies have reported that TRIM24 is a secondary coactivator, which indirectly activates NRs in cooperation with several coactivators (Teyssier et al., 2006). To evaluate this possibility for the CAR, we investigated the cooperative effects of TRIM24 with SRC1 and SRC2, which are known coactivators of the CAR. The overexpression of each coactivator increased the luciferase activity from a DR4-driven luciferase reporter plasmid in HepG2 cells expressing the CAR. Simultaneous overexpression of TRIM24 significantly and more strongly augmented the increase in luciferase activities compared with the cells overexpressing SRC1 or SRC2 alone (Fig. 4).

TRIM24 Is Corecruited with the CAR to the PBREM Region of the CYP2B6 Promoter. Next, we carried out a ChIP analysis to determine whether TRIM24 was corecruited with the CAR to the PBREM region of the CYP2B6 promoter containing a CAR-response element. We used HepTR/hCAR cells, which are HepG 2 cells carrying a Tet-inducible CAR gene. The ChIP analysis revealed that the CAR and TRIM24 both recruited to the PBREM region in the presence of Tet treatment (Fig. 5). This result indicated that TRIM24 was recruited with the CAR to the PBREM region.

TRIM24 Is an Important Factor for the Promoter-Selective Transactivity of the CAR. The effects of TRIM24 on the CARinduced expression of CYP2B6 and CYP3A4 genes were also assessed following TRIM24 knockdown in HepTR/hCAR cells. In the HepTR/hCAR cells, the knockdown of TRIM24 by siRNA and overexpression of the CAR by Tet were confirmed by western blotting (Fig. 6A) and the mRNA expression of CYP2B6 and CYP3A4 mRNAs was also examined (Fig. 6B). Knocking down TRIM24 suppressed Tet-inducible CYP2B6 mRNA expression, but did not affect CYP3A4 mRNA expression. We further investigated the effects of TRIM2 4 on the CAR-inducible mRNA expression using HepaRG cells as a highly differentiated model for studies of the CAR activation. Treatment with CITCO, an agonist of the CAR increased the CYP2B6 and CYP3A4 mRNA expression (Fig. 7). The induction of CYP2B6 and CYP3A4 mRNA by CITCO was suppressed by TRIM24 knockdown, although CYP3A4 showed downregulated basal expression (in the absence of CITCO).

\section{Discussion}

The identification and characterization of coregulators that modulate individual NRs are important to understand the physiologic functions of the NRs. The NR CAR plays an important role in xenobiotic/drug metabolism. We previously identified DP97 and protein arginine methyltransferase 5 as gene (or promoter)-selective CAR coactivators (Kanno et al., 2012, 2015). In the present study, we showed that knocking down TRIM24 reduced the CAR-mediated upregulation of CYP2B6 mRNA, whereas the CAR-mediated upregulation of CYP3A4 mRNA was insensitive to TRIM24 knockdown in HepTR/CAR cells. Similarly, it was observed that knocking down TRIM24 reduced the CITCOmediated CYP2B6 mRNA induction in HepaRG cells. Although the CITCO-treated group of CYP3A4 mRNA was decreased by TRIM24 knockdown in HepaRG cells, fold response was not suppressed. These 
results suggest that TRIM24 is a novel coactivator of the CAR, which is involved in cell- and/or promoter-selective transactivation.

Previous reports showed that TRIM24 associated simultaneously with SRC2 and coactivator-associated arginine methyltransferase 1, and synergic transcriptional activation of the glucocorticoid receptor was observed with these coactivators (Teyssier et al., 2006). Because TRIM24 apparently lacks a transcriptional activation function, the role of TRIM24 was unknown. It was reported that the plant homeodomain/bromodomain region of TRIM24 acts as a reader of dual histone marks (Tsai et al., 2010). TRIM24 recognizes unmodified histone $\mathrm{H} 3$ at lysine $4(\mathrm{H} 3 \mathrm{~K} 4)$ and acetylated histone 3 at lysine 23 (H3K23ac) within the same histone tail. It was suggested that this function as a histone mark reader is important for coactivation. The CAR-binding enhancer element of CYP2B6 at -1800 base pairs, called the PBREM, contains two DR4 elements (NR1 and NR2) that act as high-affinity binding sites for the CAR/retinoid X receptor $\alpha$ (Sueyoshi et al., 1999). CYP3A4 also has a CAR-binding enhancer element at -7800 base pairs, called the xenobiotic responsive enhancer module, which contains the distal direct repeat 3-type NR-binding element (dNR1) and the DR4-type essential distal NR-binding element for CYP3A4 induction (eNR3A4) (Goodwin et al., 1999; Toriyabe et al., 2009). However, whether there is histone modification in these enhancer elements upon CAR-mediated transactivation is unknown. The difference in TRIM24-mediated coactivation between CYP2B6 and CYP3A4 might be due to differences in the histone marks in these enhancer elements. Further investigation into histone modification by CAR is required to understand the TRIM24-selective coactivation.

TRIM24 was shown to suppress tumorigenesis during retinoic acid receptor activation to prevent liver cancer in a murine model. The tripartite motif transcription cofactors, TRIM24, TRIM28, and TRIM33, associate to form regulatory complexes that repress murine hepatocellular carcinoma (Khetchoumian et al., 2007; Herquel et al., 2011). Furthermore, TRIM24 knockout mice spontaneously develop hepatic lipidfilled lesions, steatosis, hepatic injury, fibrosis, and hepatic carcinoma since TRIM24 suppresses hepatic lipid accumulation, inflammation, fibrosis, and damage (Jain et al., 2014; Jiang et al., 2015). Because there is increasing evidence that the CAR is involved in energy metabolism and tumorigenesis, the relationship between the CAR and TRIM24 with regard to energy metabolism and tumorigenesis should be further analyzed.

In conclusion, we herein demonstrated that TRIM24 is a novel coactivator of the CAR, which acts synergistically with SRC1 and/or SRC2 to enhance CAR-mediated transcription. Furthermore, TRIM24 regulates the promoter-selective coactivation of target genes. TRIM24 may therefore be a key regulator of selective gene induction by the CAR. However, further investigations are required to understand the selective regulation mechanism.

\section{Authorship Contributions}

Participated in research design: Kanno, Inouye.

Conducted experiments: Kanno, Kure, Kobayashi, Mizuno, Yamashita, Tsuchiya.

Contributed new reagents or analytic tools: Kanno.

Performed data analysis: Kanno, Kure, Kobayashi, Mizuno, Yamashita, Tsuchiya.

Wrote or contributed to the writing of the manuscript: Kanno, Nemoto, Inouye.

\section{References}

Baes M, Gulick T, Choi HS, Martinoli MG, Simha D, and Moore DD (1994) A new orphan member of the nuclear hormone receptor superfamily that interacts with a subset of retinoic acid response elements. Mol Cell Biol 14:1544-1552.

Chen T, Chen Q, Xu Y, Zhou Q, Zhu J, Zhang H, Wu Q, Xu J, and Yu C (2012) SRC-3 is required for CAR-regulated hepatocyte proliferation and drug metabolism. J Hepatol 56:210-217.

Choi E, Lee S, Yeom SY, Kim GH, Lee JW, and Kim SW (2005) Characterization of activating signal cointegrator-2 as a novel transcriptional coactivator of the xenobiotic nuclear receptor constitutive androstane receptor. Mol Endocrinol 19:1711-1719.
Goodwin B, Hodgson E, and Liddle C (1999) The orphan human pregnane X receptor mediates the transcriptional activation of CYP3A4 by rifampicin through a distal enhancer module. $\mathrm{Mol}$ Pharmacol 56:1329-1339.

Gronemeyer H, Gustafsson JA, and Laudet V (2004) Principles for modulation of the nuclear receptor superfamily. Nat Rev Drug Discov 3:950-964.

Herquel B, Ouararhni K, Khetchoumian K, Ignat M, Teletin M, Mark M, Béchade G, Van Dorsselaer A, Sanglier-Cianférani S, Hamiche A, et al. (2011) Transcription cofactors TRIM24, TRIM28, and TRIM33 associate to form regulatory complexes that suppress murine hepatocellular carcinoma. Proc Natl Acad Sci USA 108:8212-8217.

Honkakoski P, Zelko I, Sueyoshi T, and Negishi M (1998) The nuclear orphan receptor CAR-retinoid $X$ receptor heterodimer activates the phenobarbital-responsive enhancer module of the CYP2B gene. Mol Cell Biol 18:5652-5658.

Jain AK, Allton K, Duncan AD, and Barton MC (2014) TRIM24 is a p53-induced E3-ubiquitin ligase that undergoes ATM-mediated phosphorylation and autodegradation during DNA damage. Mol Cell Biol 34:2695-2709.

Jiang S, Minter LC, Stratton SA, Yang P, Abbas HA, Akdemir ZC, Pant V, Post S, Gagea M, Lee RG, et al. (2015) TRIM24 suppresses development of spontaneous hepatic lipid accumulation and hepatocellular carcinoma in mice. J Hepatol 62:371-379.

Jyrkkärinne J, Küblbeck J, Pulkkinen J, Honkakoski P, Laatikainen R, Poso A, and Laitinen T (2012) Molecular dynamics simulations for human CAR inverse agonists. J Chem Inf Model 52: $457-464$.

Kanno Y, Inajima J, Kato S, Matsumoto M, Tokumoto C, Kure Y, and Inouye Y (2015) Protein arginine methyltransferase 5 (PRMT5) is a novel coactivator of constitutive androstane receptor (CAR). Biochem Biophys Res Commun 459:143-147.

Kanno Y and Inouye Y (2010) A consecutive three alanine residue insertion mutant of human CAR: a novel CAR ligand screening system in HepG2 cells. J Toxicol Sci 35:515-525.

Kanno Y, Serikawa T, Inajima J, and Inouye Y (2012) DP97, a DEAD box DNA/RNA helicase, is a target gene-selective co-regulator of the constitutive androstane receptor. Biochem Biophys Res Commun 426:38-42.

Kanno Y, Suzuki M, Miyazaki Y, Matsuzaki M, Nakahama T, Kurose K, Sawada J, and Inouye Y (2007) Difference in nucleocytoplasmic shuttling sequences of rat and human constitutive active/androstane receptor. Biochim Biophys Acta 1773:934-944.

Kanno Y, Suzuki M, Nakahama T, and Inouye Y (2005) Characterization of nuclear localization signals and cytoplasmic retention region in the nuclear receptor CAR. Biochim Biophys Acto 1745:215-222.

Kawamoto T, Sueyoshi T, Zelko I, Moore R, Washburn K, and Negishi M (1999) Phenobarbitalresponsive nuclear translocation of the receptor CAR in induction of the CYP2B gene. Mol Cell Biol 19:6318-6322.

Khetchoumian K, Teletin M, Tisserand J, Mark M, Herquel B, Ignat M, Zucman-Rossi J, Cammas F, Lerouge T, Thibault C, et al. (2007) Loss of Trim 24 (Tifl $\alpha$ ) gene function confers oncogenic activity to retinoic acid receptor alpha. Nat Genet 39:1500-1506.

Kikuchi M, Okumura F, Tsukiyama T, Watanabe M, Miyajima N, Tanaka J, Imamura M, and Hatakeyama S (2009) TRIM24 mediates ligand-dependent activation of androgen receptor and is repressed by a bromodomain-containing protein, BRD7, in prostate cancer cells. Biochim Biophys Acta 1793:1828-1836.

Le Douarin B, Nielsen AL, Garnier JM, Ichinose H, Jeanmougin F, Losson R, and Chambon P (1996) A possible involvement of TIF1 alpha and TIF1 beta in the epigenetic control of transcription by nuclear receptors. EMBO J 15:6701-6715.

Le Douarin B, Zechel C, Garnier JM, Lutz Y, Tora L, Pierrat P, Heery D, Gronemeyer H, Chambon $\mathrm{P}$, and Losson R (1995) The N-terminal part of TIF1, a putative mediator of the ligand-dependent activation function (AF-2) of nuclear receptors, is fused to B-raf in the oncogenic protein T18. EMBO J 14:2020-2033.

Mangelsdorf DJ, Thummel C, Beato M, Herrlich P, Schütz G, Umesono K, Blumberg B, Kastner P, Mark M, Chambon P, et al. (1995) The nuclear receptor superfamily: the second decade. Cell 83:835-839.

Min G, Kemper JK, and Kemper B (2002) Glucocorticoid receptor-interacting protein 1 mediates ligand-independent nuclear translocation and activation of constitutive androstane receptor in vivo. J Biol Chem 277:26356-26363

Muangmoonchai R, Smirlis D, Wong SC, Edwards M, Phillips IR, and Shephard EA (2001) Xenobiotic induction of cytochrome P450 2B1 (CYP2B1) is mediated by the orphan nuclear receptor constitutive androstane receptor (CAR) and requires steroid co-activator 1 (SRC-1) and the transcription factor Sp1. Biochem J 355:71-78.

Shiraki T, Sakai N, Kanaya E, and Jingami H (2003) Activation of orphan nuclear constitutive androstane receptor requires subnuclear targeting by peroxisome proliferator-activated receptor gamma coactivator- $1 \alpha$. A possible link between xenobiotic response and nutritional state. $J$ Biol Chem 278:11344-11350.

Sueyoshi T, Kawamoto T, Zelko I, Honkakoski P, and Negishi M (1999) The repressed nuclear receptor CAR responds to phenobarbital in activating the human CYP2B6 gene. J Biol Chem 274:6043-6046.

Sugatani J, Kojima H, Ueda A, Kakizaki S, Yoshinari K, Gong QH, Owens IS, Negishi M, and Sueyoshi T (2001) The phenobarbital response enhancer module in the human bilirubin UDP-glucuronosyltransferase UGT1A1 gene and regulation by the nuclear receptor CAR. Hepatology 33:1232-1238.

Surapureddi S, Rana R, Reddy JK, and Goldstein JA (2008) Nuclear receptor coactivator 6 mediates the synergistic activation of human cytochrome P-450 2C9 by the constitutive androstane receptor and hepatic nuclear factor-4alpha. Mol Pharmacol 74: 913-923.

Teyssier C, Ou CY, Khetchoumian K, Losson R, and Stallcup MR (2006) Transcriptional intermediary factor 1alpha mediates physical interaction and functional synergy between the coactivator-associated arginine methyltransferase 1 and glucocorticoid receptor-interacting protein 1 nuclear receptor coactivators. Mol Endocrinol 20: $1276-1286$.

Tian J, Huang H, Hoffman B, Liebermann DA, Ledda-Columbano GM, Columbano A, and Locker $\mathrm{J}$ (2011) Gadd45 $\beta$ is an inducible coactivator of transcription that facilitates rapid liver growth in mice. J Clin Invest 121:4491-4502.

Timsit YE and Negishi M (2014) Coordinated regulation of nuclear receptor CAR by CCRP/DNAJC7, HSP70 and the ubiquitin-proteasome system. PLoS One 9:e96092.

Toriyabe T, Nagata K, Takada T, Aratsu Y, Matsubara T, Yoshinari K, and Yamazoe Y (2009) Unveiling a new essential cis element for the transactivation of the CYP3A4 gene by xenobiotics. Mol Pharmacol 75:677-684. 
Tsai WW, Wang Z, Yiu TT, Akdemir KC, Xia W, Winter S, Tsai CY, Shi X, Schwarzer D, Plunkett W, et al. (2010) TRIM24 links a non-canonical histone signature to breast cancer. Nature 468:927-932.

vom Baur E, Zechel C, Heery D, Heine MJ, Garnier JM, Vivat V, Le Douarin B, Gronemeyer H, Chambon P, and Losson R (1996) Differential ligand-dependent interactions between the AF-2 activating domain of nuclear receptors and the putative transcriptional intermediary factors mSUG1 and TIF1. EMBO J 15:110-124.

Windshügel B, Jyrkkärinne J, Poso A, Honkakoski P, and Sippl W (2005) Molecular dynamics simulations of the human CAR ligand-binding domain: deciphering the molecular basis for constitutive activity. J Mol Model 11:69-79.
Yoshinari K, Kobayashi K, Moore R, Kawamoto T, and Negishi M (2003) Identification of the nuclear receptor CAR:HSP90 complex in mouse liver and recruitment of protein phosphatase 2A in response to phenobarbital. FEBS Lett 548:17-20.

Address correspondence to: Dr. Yuichiro Kanno, Department of Molecular Toxicology, Faculty of Pharmaceutical Sciences, Toho University, Funabashi, Chiba 274-8510, Japan. E-mail: ykanno@phar.toho-u.ac.jp 